\title{
Familias judías-familias conversas. Aproximación a los neófitos valencianos del siglo XIV
}

\author{
JosÉ LUIS LUZ COMPAÑ *
}

\section{INTRODUCCIÓN}

En 1395 los diputados de los conversos se dirigen al "Consell» de la ciudad de Valencia, solicitando que sea leída una carta real, sello secreto, dirigida a los jurados, en la que se mencionan las fuertes cargas que han de soportar para compensar las deudas de la antigua judería, "se són entre ells fetes talles $\ominus$ compartiments a rahó de set sous e sis diners per liura de tots lurs" ${ }^{1}$. La situación que transluce este documento es la de que los conversos se hallan en un momento de extrema penuria, prácticamente mendigando ayuda. Escasamente medio siglo después, poco antes del pogrom anticonverso de Toledo, las protestas por el volumen de riqueza y poder que han alcanzado los neófitos son generalizadas. ¿Qué sucedió en esos escasos 60 años para que la situación diese un giro de 180 grados? ¿Quiénes, entre el grupo de nuevos convertidos, alcanzaron esta situación de preponderancia? ¿Cuáles fueron sus estrategias? ¿Fue un fenómeno generalizado, o bien afectó sólo a una élite? Éstas son algunas de las preguntas que surgen tras una primera aproximación al estudio de los conversos antes de la actuación de la Inquisición.

Para comprender mejor este fenómeno es necesario remontarse a los inicios del «problema converso», es decir a la última década del siglo XIV, para analizar los puntos de partida reales de las nuevas familias conversas y seguir su decurso hasta mediados del siglo $\mathrm{XV}$, un período más conocido

* Universidad de Valencia.

1 Archivo Municipal de Valencia, Manuals de Consell, A-20, fol. 255r, 1395-octubre-29. VIDAL Beltrán, E., Valencia en la época de Juan I. Valencia, Universidad, 1974, pág. 71, n. 1. 
gracias a los procesos inquisitoriales. Se trata de obtener una visión global que no se centre exclusivamente en las élites, sino que debe enfocarse hacia el conjunto de la población conversa y analizar su evolución económica y social con la mayor proximidad posible, teniendo siempre en cuenta que el grupo de los conversos es tan heterogéneo en su composición y evolución como el resto de la sociedad dominante.

Bibliográficamente se trata de un período poco y superficialmente estudiado. Existen buenos trabajos, tanto a nivel del País Valenciano como peninsular, sobre los momentos anteriores al asalto y para la segunda del siglo $\mathrm{XV}^{2}$. Sin embargo, carecemos de estudios sistemáticos que aporten luz a lo que pudo suceder a lo largo de ese medio siglo. La mayoría de los autores parece coincidir en que se trata de un período de cierta tranquilidad social y de dinámico crecimiento económico, durante el cual los conversos fueron ascendiendo en la escala socioeconómica a la par que sus hermanos judíos entraban, con más o menos celeridad, en una profunda decadencia. Declive que coincidiría con el momento de auge de los nuevos convertidos, para acabar, en ambos casos, trágicamente y casi de forma simultánea. A lo largo del período de relativa tranquilidad social que podríamos enmarcar entre los años 1392 y 1449, solamente los judíos se vieron afectados por los últimos coletazos del movimiento antisemita eclosionado en 1391. La Disputa de Tortosa (1413-1415), vino a significar el fin del ciclo de las conversiones y el inicio de una nueva época en las relaciones entre los descendientes del pueblo hebreo y la sociedad cristiana dominante ${ }^{3}$.

Las líneas de investigación más novedosas han llamado la atención sobre la importancia del papel de la familia, los lazos de parentesco y las

2 Aparte de las ya clásicas sintesis de Amador de los Ríos y Yitzak Baer, existen todo un variado elenco de estudios refericios a los judios valencianos que sería muy largo, y excluitorio, reflejar aquí. Por mencionar algunos referidos al País Valenciano, son de gran interés los trabajos del recientemente malogrado Leopoldo Piles, que destacan por su amplio aporte documental, así como los de José Hinojosa Montalvo y Angelina García Pérez sobre las actividades económicas de los judíos valencianos durante los siglos bajomedievales. También hay que tomar en cuenta las interesantes aportaciones de José Ramón Magdalena para los judíos castellonenses, sobre todo a lo largo del siglo xv. Tampoco hay que dejar de lado las aportaciones, si bien puntuales, de otros autores entre los que cabría destacar a David Romano. Para una información bibliográfica más exhaustiva, véase el reciente artículo de HINOJOSA, «En torno a los judíos valencianos: la recuperación de una minoría olvidada», Hispania, L/2, n. ${ }^{\circ} 175,1990$, págs. $921-940$

Respecto a los conversos valencianos, el número de obras es particularmente escaso, si bien conviene destacar García PÉrez, A., Els Vives: Una familia de jueus valencians. Valencia, Ed. Tres i Quatre,1987.

3 Sobre el tema de la Disputa de Tortosa, vid. PACios López, A., La Disputa de Tortosa, CSIC e Instituto Arias Montano, 2 vols. Madrid 1957. 
formas de solidaridad para el mejor conocimiento de los comportamientos económicos, sociales y mentales, de una sociedad dada. Para darse cuenta del éxito que han tenido estas nuevas corrientes de investigación, no hay más que ver el amplio volumen de publicaciones que tratan el tema y las mutaciones metodológicas que reflejan los diferentes estudios sectoriales que se han desarrollado desde la década de los ochenta ${ }^{4}$. A partir de las nuevas perspectivas abiertas por la historiografía, y los buenos resultados obtenidos, pensamos que el análisis de la evolución del grupo converso debe enfocarse tomando como base el ámbito familiar. Observar la fenomenología de los comportamientos que adopta el grupo de los neófitos valencianos frente al reto que supone la integración, es decir, la «indiferenciación», dentro de la sociedad cristiana; en resumen, la delimitación de las transformaciones sufridas por el «pueblo de los conversos» a lo largo del «proceso de adaptación» a las nuevas circunstancias, ha de pasar por el estudio detallado de la célula familiar.

Existe otro motivo para enmarcar el estudio en torno a la familia. Con el tiempo, los conversos tienden a enmarcarse, a ocultarse en los pliegues de la sociedad dominante, por ello el historiador debe proceder a un proceso de «identificación» que sólo puede ser realizado a través de prosopografías que emanan, naturalmente, a partir del estudio de la familia. La reconstrucción prosopográfica nos permitirá también realizar un seguimiento puntual y detallado de la evolución socioeconómica conversa, a la vez que analizamos la heterogeneidad del grupo, y las diferentes estrategias desarrolladas por los diversos estratos del colectivo de neófitos valencianos, para luego tratar de llegar a una síntesis que sea capaz de aglutinar las diferentes variables, tanto sociales y económicas como mentales, que definirán el despegue converso desde el furor antisemita de 1391 hasta el inicio de las persecuciones inquisitoriales.

Paralelamente, en nuestra investigación pretendemos estudiar la comunidad conversa, no como una realidad aparte, como un grupo autónomo que se rige por principios distintos a los de la sociedad circundante, sino que, por el contrario, pensamos que la presente investigación debe estar dominada por un afán integrador. El «pueblo de los conversos» debe ser observado como parte activa de la sociedad valenciana bajomedieval. El conjunto documental que hemos estudiado hasta el momento, tanto para judíos valencianos como para conversos, confirma que, pese a tra-

4 Por citar dos ejemplos de obras de síntesis sobre el tema podríamos destacar BuRGuIERE, A. et alii, Historia de la Familia. Madrid, Alianza, 1988; y Arıes, Ph. y DuBY, G., Historia de la vida privada. Madrid, Taurus, 1988. 
tarse de un grupo con características bastante definidas, sus actividades se hallan perfectamente integradas dentro de la dinámica social de la época, de manera que resultaría imposible llegar a conclusiones certeras, tanto en el estudio de la población hebrea como de la cristiana, sin tener en cuenta el papel desempeñado por judíos y conversos como dos células más dentro del tejido social bajomedieval.

Era necesario buscar, pues, nuevas fuentes que nos permitieran una aproximación más directa a las actividades de judíos y conversos. Un acercamiento que debía contemplar el conjunto y no solamente hacer incapié en las élites económicas que adquieren un papel importante en las instituciones. Las fuentes documentales que nos han servido de base son los protocolos notariales, muy abundantes para la Valencia medieval, sobre todo desde el comienzo del trescientos. Documentos que hasta hoy en día han sido muy poco aprovechados por la historiografía y que se nos presentan muy útiles a la hora de estudiar el comportamiento individual y el papel del grupo familiar en las diversas situaciones, tanto públicas como privadas. Las actividades económicas, las variaciones del comportamiento individual o colectivo, aparecen con gran fiabilidad, despojados de la tendenciosidad atribuible a la documentación emanada de las instituciones o de los modelos sociales segregados por las clases dominantes, en los contratos de dote, de comanda, aprendizaje y servicio, testamentos o inventarios, cartas de tutela o procuración, títulos de deuda, etc. Con estas bases documentales, el grupo social objeto de nuestro estuaio, presentaba una fácil identificación -en un primer momento la palabra conversus acompaña siempre al nombre-, haciendo más asequible su seguimiento. Paralelamente, la documentación notarial, proporciona bastantes facilidades para un análisis informatizado, por lo que, teniendo en cuenta los objetivos de nuestra investigación, quedaba convertida en una fuente privilegiada para su consecución. En este sentido nos han sido muy útiles los protocolos del notario Berthomeu de la Mata, conservados en el Archivo del Reino de Valencia y en el Archivo de Protocolos del Patriarca de Valencia, que presentan una serie bastante continua desde 1386 hasta 1415 , por lo que los hemos tomado como base del presente trabajo ${ }^{5}$.

\section{LA DINÁMICA ECONÓMICA}

Como hemos dicho al principio, tras el asalto, la situación económica de los conversos se nos presenta próxima a la catástrofe. Muchos de

5 Queremos agradecer aquí la ayuda en la localización de estas fuentes a los compañeros del grupo de trabajo para el estudio de la sociedad urbana bajomedieval dirigido por el Dr. Iradiel. 
ellos han perdido una parte de sus bienes, no solamente el dinero líquido - los objetos valiosos de fácil transporte, sino también han visto destruidos - arrebatados sus medios de producción ${ }^{6}$. En medio de aquella euforia milenarista, sus lugares de culto y sus libros de oración han sido profanados y, en algunos casos, destruidos. Prácticamente todos los documentos encontrados en casas de judíos, fundamentalmente los escritos en hebreo, desaparecieron, y entre ellos cientos de títulos de deuda, juntamente con contratos de dote, testamentos, etc.

Pero la descripción catastrofista emanada de las fuentes institucionales no debe conducirnos a error. La documentación que hemos recogido hasta el momento no refleja ni una situación de pánico ni, tampoco, una euforia liquidacionista, sino, más bien, una prudente cautela frente al nuevo estado de cosas. Prudencia que revela la idea de una política de reconstrucción, aparentemente bastante meditada, que tratará de recuperar el pulso económico de las familias judeoconversas y que obtendrá sus primeros frutos en la segunda mitad de la década.

Como primera medida, se buscará la recuperación del mayor número posible de títulos de deuda. De los más de 400 títulos que poseemos para los primeros años posteriores a 1391, casi un centenar, corresponden a reconocimientos de deuda (reconeiximents de deute). Por ejemplo, en enero de 1392 Anthoni Martí, agricultor comorante en Catarroja, reconoce deber al converso Manuel Dez Puig, antes Maymo Alicení, habitante de Valencia, la cantidad de 60 sueldos, deuda que se remontaba a $1386^{7}$. Paralelamente, puesto que los títulos de deuda no siempre eran fáciles de ejecutar, para recapitalizarse se recurre al «carregament» de censales o violarios, o bien al simple préstamo, vendidos en principio a aquellos conversos que mantenían una cierta reserva de capital, como se da en el caso del converso Bernat Andreu, antes Azdra Cresques, mercader, quien presta a Nicholau Salvador, antes Salamó Alicení, 12 florines a devolver en ocho meses, a razón de un florín y medio cada mes ${ }^{8}$. Todavía no podemos afirmar el alcance que tuvo la política de endeudamiento practicada por los conversos frente a otras opciones como la liquidación de bienes, si bien parece evidente que un número notable de conversos recurrió al mercado del crédito para reflotar, en un primer momento, su economía. Podría ser el caso de Pasqual Maçana, antes Naçan Abenmarueç, quien junto con otro converso, Pere de Muncada, y un judío,

6 Vidal Beltrán, E., Valencia en la época de Juan I. Valencia, Universidad, 1974, sobre todo págs. 51-74.

Archivo del Reino de Valencia (en adelante ARV), pág. 1444, 1392-enero-2.

a Idem, 1392-enero-9. 
Salamó Naçí, pagan una renta anual de 500 sueldos a María Marrades, viuda de Francesch Marrades, ciudadano de Valencia ${ }^{9}$. El caso de Pasqual Maçana es sintomático de lo que sugerimos. Sus actividades económicas anteriores al asalto parecen hallarse muy centradas en el mercado del crédito $y$, seis meses después del mismo, no parecen haber decaído en exceso. Solamente durante el mes de enero de 1392 adquirirá títulos de deuda por un valor de 2.095 sueldos, lo que nos da a entender que su situación económica no era precisamente precaria. Otro caso similar es el del físico judío lçach Abenazrá, de converso Johan Torregrosa, quien a lo largo del año 1392 continúa comprando censales y violarios a un ritmo muy similar al anterior al 9 de julio de 1391.

La ausencia de enajenaciones en la documentación nos induce a pensar que en un primer momento no se dio un proceso de fuerte desposesión de bienes inmobilarios como consecuencia del asalto, aunque algunos neófitos consideraron prudente apartarse de la judería durante un tiempo y trasladarse a otros puntos de la ciudad, para regresar a la misma algunos años después. No obstante, la mayoría de los conversos optaron por permanecer en la antigua judería trabajando juntos y tratando de recuperar las antiguas solidaridades violentamente truncadas.

En resumen, si bien es cierto que la información que manejamos es todavía escasa para realizar afirmaciones certeras, lo que hasta el momento hemos observado es una rápida reconstrucción inicial, hasta unos niveles medios, de la tasa de riqueza de algunas de las familias conversas, y pensamos que se puede hablar de recuperación generalizada a partir de 1395, cuando el relanzamiento de la economía del grupo de cristianos nuevos se nos manifiesta ya como evidente.

En cuanto a las actividades profesionales, los conversos aparecen muy relacionados tanto con la esfera de la producción como con la de la circulación. Siempre se ha asociado a los judíos primero y a los conversos después con oficios de marcado carácter comercial. Evidencias documentales de ello no faltan, existe un amplio volumen de conversos que se definen como «curritor», "mediator» o «mercator».

Desde la década de los años 80 del siglo xIV se detecta una evolución importante, que se tornará sorprendentemente rápida tras el asalto. Con anterioridad no hemos encontrado ningún judío en la documentación que se defina como mercader, la gran mayoría afirman ser corredores de comercio, tarea que compatibilizan con el oficio correspondiente, ya sea el

9 ARV, pág. 10407, 1397-julio-15. 
de sastre, platero u otro cualquiera. El cierto grado de cualificación que surge de un amplio espectro de conexiones sociales, emanadas de las relaciones laborales (proveedores, clientes, etc.), de unas afinidades familiares y religiosas diversificadas y de la dedicación parcial al crédito, hacen de los hebreos unos estupendos conocedores del mercado.

A partir de 1391, las cosas parecen cambiar. Comienza a detectarse una mutación, no sólo en el ámbito económico, sino también en la esfera de lo social. Muchos conversos aparecen desempeñando un papel muy activo en las relaciones comerciales a la vez que socialmente alcanzan el estatuto de «vehïns», que anteriormente, como judíos, les había estado vedado. Y no sólo eso, poco después, algunos de ellos conseguirán el estatuto de ciudadanos, es decir el disfrute de plenos derechos políticos.

La eclosión se producirá en la segunda mitad de la década. Para esta época la documentación revela una gran actividad productiva. Numerosos conversos están especializados en la producción textil. Aparecen una gran cantidad de documentos, algunos de ellos datan de 1392, de compra de materias primas y venta de productos acabados. Las actividades manufactureras de los conversos parecen especializarse en el trabajo de la seda, a juzgar por el gran volumen de adquisiciones de este producto en el que intervienen. En 1393, Johan Catalá, converso, compra 28 libras de seda a Nicholau Marrades, antes Samuel Mercadell, por valor de 30 florínes de oro ${ }^{10}$; al igual que posteriormente en 1399 encontramos al mercader Gabriel Vives, ciudadano de Valencia, converso, adquiriendo 16 libras de seda tintada, por un precio conjunto de 37 libras y tres sueldos, a un proveedor habitual, Jaume Mercer ${ }^{11}$. También comerciarán con otras materias primas como el lino y la lana. Los artesanos conversos aparecen con cierta frecuencia vendiendo productos elaborados, como el pelaire Johan de Caldes, que actúa con gran actividad a partir de 1397. En general, todo el sector productivo se halla en movimiento, se incrementa el número de contratos de aprendizaje y trabajo. Otros documentos corroboran indirectamente estas afirmaciones como el incremento de compromisos por parte de artesanos de no dedicarse al juego durante un período de tiempo que oscila entre los dos y cinco años, de lo que deducimos que entre ellos existe algún tipo de relación laboral. El documento sanciona una toma de responsabilidad recíproca por parte de los participantes.

10 ARV, pág. 3241, 1393-enero-9.

1 ARV, pág. 1445, 1399-febrero-12. 
Hay indicios claros de que algunas de las grandes fortunas de la judería han finalizado una primera fase de recuperación, sus actividades se diversifican y amplían a través de las reagrupaciones familiares y la formación de asociaciones comerciales. A partir de 1399 comienzan a aparecer documentos que atestiguan la formación de compañías laborales y comerciales, como la mantenida entre Francesch Castellar y Johan VaIleriola, sastres de Valencia, y que data, seguramente, de años anteriores $^{12}$; o la constituida por Pere Dartés, sedero, y Pere Dauder, mercader, "super arte mercatorie». Dartés se compromete a invertir 111 libras y Pere Dauder habrá de invertir 46 libras y encargarse de viajar a Zaragoza a vender los productos, si bien ignoramos a qué tipo de mercado iban dirigidos ${ }^{13}$. Paralelamente en los protocolos comienzan a aparecer un número importante de conversos que se califican como «mercator». El nombramiento de procuradores encargados de gestionar las mercancías aumenta. Hallamos cartas de procuración referidas a agentes situados a lo largo de toda la Corona de Aragón. En 1397, Johan Catalá, mercader de Valencia, nombra procurador a Gabriel del Mas, para que gestione algunas mercancías en Aragón ${ }^{14}$; en agosto de 1399, Johan Rossell de Mijavila, mercader y ciudadano de Valencia, nombra procurador suyo a Gabriel Dezpuig, también comerciante y ciudadano, para que recupere una cantidad retenida por Guillem de Salmons, mercader de Barcelona, factor suyo en dicha ciudad ${ }^{15}$. Algunos de estos procuradores serán judíos, a menudo están relacionados familiarmente, tal es el caso de Manuel Salvador, antes Mahir Suxen, ciudadano de Xátiva, que nombra procurador a su hermano Samuel Suxen, rico financiero de Sagunt, para que cobre en su nombre algunas deudas ${ }^{16}$. Con frecuencia, miembros de una misma familia conversa desplazan su residencia a otros lugares para abarcar un espacio económico más amplio. Sirva el ejemplo de la familia Rafael, cuyos miembros se hallan ubicados en Xátiva y en Valencia, manteniendo relaciones comerciales constantes entre ambos puntos.

El mercado de la tierra y los productos agrarios tampoco es ajeno a las actividades de los conversos. Lejos de emplear tan sólo el crédito agrario como forma de obiener una renta proviniente de la comercialización de los productos rurales, se adentran en la esfera de la producción. En 1399, Jaume Angless, corredor, vecino de Valencia, paga a Anthoni

Idem, 1399-junio-26.

Idem, 1399-septiembre-1.

ARV, pág. 10407, 1397-mayo-9.

ARV, pág. 1445, 1399-agosto-30

ARV, pág. 10407, 1397-noviembre-22. 
Auleger, residente en Albalat, localidad próxima a Valencia, la cantidad de ocho libras por el trabajo de mantenimiento de unas viñas que el primero posee en el término de dicha localidad. Poco después otro documento nos informa de un pago de similares características a Jaume Roig, vecino de Museros. A finales de ese mismo año, Angles arrienda "a miges" ocho anegadas de viña que tiene en el término de Albalat ${ }^{17}$. En algunos momentos también les encontramos vendiendo tierra. En 1397, Gilabert Dartés, ciudadano de Valencia, converso, vende a Hamet Almuçeniç, sarraceno de Ribarroja, dos tahullas de tierra situadas en la huerta de dicho lugar ${ }^{18}$. Tampoco dejarán de lado la integración en el mercado de los productos agrícolas. Hemos detectado conversos actuando en compraventas de grano, madera, vendimia con destino a la fabricación de vinos, etc.

El mercado del crédito sigue desempeñando un papel de cierta importancia entre las actividades económicas de los conversos. El volumen de derechos de cobro adquiridos no solamente se mantiene, sino que se incrementa sufriendo, paralelamente, una mutación. En un principio, los judíos prefieren el sistema de préstamo ${ }^{19}$, pero a partir de la década de los noventa, dedicarán sus esfuerzos en este sentido a la adquisición de rentas, es decir, a la compra de censales y violarios a tiempo indefinido, que generan una renta, según hemos podido comprobar, en torno al 14,2 por 100 anual, lo que implicaba un mayor beneficio que el préstamo a seis meses o un año, aunque estos últimos rentaran, oficialmente al menos, un 20 por 100. De este modo, Pasqual Maçana que invierte 1.966 sueldos en la compra de estos títulos a lo largo de 1399, obtendría unos derechos de cobro anuales por valor mínimo de 279 sueldos, sin contar los derechos acumulados de años anteriores. No es un caso único, muchos conversos invierten parte de sus ganancias en la compra de rentas. Aunque si bien la dedicación al crédito no es una actividad marginal dentro de la economía de los cristianos nuevos, no tiene tampoco el peso específico que se le ha querido dar $\in n$ algunos momentos. Los conversos parecen haber enfocado la adquisición de títulos de deuda más como una reserva de capital en forma de renta o, en algunos casos, como forma de mediatización de la producción. Otra hipótesis circunstancial al respecto sería la formación de clientelas a través de los vínculos financieros,

ARV, pág. 1445, 1399-marzo-2, marzo-29 y octubre-9.

8 ARV, pág. 10407, 1397-agosto-4.

19 Vid. Hinojosa Montalvo, J., "El préstamo judío en la ciuda de Valencia en la segunda mitad del siglo XIV", Sefarad, XLV, 1985, págs. 315-338 y GaRcía PÉrEZ, A., "El préstamo judío en la Huerta de Valencia durante el siglo XIV ", Annals, 1982, págs. 183-223. 
pero con la documentación que poseemos hasta el momento no podemos todavía enunciar opinión alguna sobre este tema.

En algunos casos particulares, como el de la familia Dartés, la compra de rentas adquiere un papel preponderante. Gilabert Dartés tiene arrendadas las rentas de Ribarroja, feudo de la familia Centelles. En 1399, Ramoneta de Centelles reconoce que ha existido un retraso en el pago de los derechos de Ribarroja a Gilabert Dartés, comprometiéndose a pagar los 600 sueldos que restan por las cantidades debidas al año anterior, 1398, y a liquidar los atrasos de años anteriores en breve ${ }^{20}$. Paralelamente, gran parte de los habitantes de Ribarroja tienen deudas particulares con algunos de los miembros de la familia Dartés, a quienes además, en algunos casos, compran cereales y otros productos necesarios. Sin embargo, se trata de un caso sumamente inusual. El mapa del crédito converso se extiende a la gran mayoría de los pueblos que circundan la capital, pero en ningún momento, al menos según la información de primera mano que poseemos, alcanzarán un grado de control similar al que pueda ejercer la familia Artés en el caso concreto de Ribarroja.

\section{LA EVOLUCIÓN SOCIAL}

Si la reconstrucción de las actividades económicas a través de los prołocolos notariales resulta un proceso sumamente complejo por las necesidades de serialización y la disparidad de tipologías documentales, tampoco resulta sencilla, en absoluto, la delimitación de la situación social de los conversos en la última década del siglo xiv. En el presente trabajo no vamos a proceder a realizar un análisis detallado de la estructuración familiar y el encuadramiento social, ya que requeriría un estudio mucho más amplio y complejo que excedería los límites de la presente comunicación. Sin embargo creemos necesario ofrecer una somera visión global fruto de un primer análisis de la documentación notarial. Documentación, que posibilita una aproximación importante a la actividad cotidiana, vulnerando su carácter eminentemente privado.

Angelina García, al hablarnos del entorno social en que hubieron de vivir las primeras generaciones de conversos, nos describe un panorama de temores y desconfianzas por ambas partes. Los cristianos viejos desconfían de las conversiones forzosas a las que se han visto sometidos los judíos valencianos. Ahora serán más difíciles de distinguir e imposibles

20 ARV, pág. 1445, 1399-abril-4. 
de aislar y limitar, ya no les aparece vedado el acceso a cargos públicos, ni la consecución de derechos políticos. No tienen, en principio mayores limitaciones a su actuación que el resto de la sociedad, con la sola excepción de los mudéjares. Conocen la facultad emprendedora que caracteriza al pueblo judío, su capacidad de supervivencia incluso en los ambientes más hostiles, al fin y al cabo 1391 no había sido el único gran pogrom antijudaico a lo largo de la centuria. La evolución económica de los conversos, su rápida recuperación hasta unos niveles medios de riqueza, parecía darles la razón. Paralelamente, el «pueblo de los conversos" responde a esta situación con una desconfianza prudente. Nada indica que las conversiones les hayan invulnerabilizado frente a los desenfrenos de la masa y ante ciertos comportamientos, menos viscerales y más políticos de ciertas capas de las élites gobernantes. El temor a un retorno a coyunturas anteriores parece no dejarles otra salida que la huida hacia adelante, es decir la renuncia a volver a practicar abiertamente su antigua religión y la apuesta por el mantenimiento externo de unos valores cristianos y unas prácticas sociales integradoras dentro de los límites de la publicidad. Angelina García vas más lejos cuando afirma que la única manera que veían los nuevos convertidos de evitar una vuelta al estatus anterior era la del control de cargos políticos y la del poder económico, en resumen, la compra y doblegación de las voluntades ${ }^{21}$. Sin embargo, el motín anticonverso de 1449 en Toledo, parece haberles quitado la razón y quizá, ese mismo hipotético deseo colectivo de riqueza y poder les habría hecho, según más de un autor, víctimas propiciatorias de la Inquisición.

Por lo que hemos podido observar, es sumamente difícil esclarecer si existe un proyecto corporativo y coordinado de inserción sistemática en los cuadros administrativos, o un afán de riqueza superior al que pudiera desarrollar cualquier otro colectivo de la Valencia bajomedieval. La idea del ascenso social no tiene por qué ser privativa de un grupo determinado; las constantes luchas de los bandos urbanos a lo largo del siglo XI v por apoderarse del poder y, por tanto, de los mecanismos de generación y distribución de la riqueza, da prueba de ello ${ }^{22}$. De hecho, el furor antijudío y posteriormente anticonverso, encuentra una de sus claves explicativas, como ha señalado Iradiel ${ }^{23}$, en la conflictividad interna del equipo patricio,

\footnotetext{
21 García, A., Els Vives..., sobre todo el cap. III.

2. Narbona Vizcaino, R., Malhechores, violencia y justicia ciudadana en la Valencia bajomedieval. Valencia, Ayuntamiento, 1990.

23 IRADIEL, P., «La crisis medieval», en Historia de España. Barcelona, Planeta, 1988, sobre todo págs. $166-188$.
} 
a cuya adscripción clientelar no serán ajenos ni los judíos ni, más tarde, los conversos. Con ello pretendemos plantear que los comportamientos integradores y de ascenso social, ni comenzarían en el momento de las conversiones, ni serían, en modo alguno, privativos del grupo de los neófitos, que representarían una fuerza más dentro de la dinámica sociopolítica urbana bajomedieval. El estudio del papel de los padrinos, tarea que aún no hemos comenzado, probablemente aclarará algunas de las hipótesis que hemos aventurado y proporcionará nuevos puntos de vista para el estudio de la situación social de los conversos.

No resultaría cierto afirmar que el colectivo de neofiti in fidem, pasó inadvertido en sus comportamientos. La sociedad medieval general mecanismos segregacionistas de forma constante, mecanismos de exclusión que no sólo afectarán al grupo estudiado, sino a toda una variada serie de comunidades a las que, por multitud de causas que sería muy largo analizar aquí, considera ajenas a su núcleo; sería el caso de los mudéjares, los mercaderes extranjeros, los marginados, etc. Los conversos verán impedida su entrada en las corporaciones gremiales, en las instituciones de ayuda mutua y el acceso de algunos de ellos a cargos de cierta relevancia no dejarán de suscitar protestas más o menos violentas. $\mathrm{Pa}$ ralelamente, aparecerán inducciones disgregacionistas que pretenden truncar las solidaridades internas del grupo, cuya más clara expresión sería la orden del «Consell» de la ciudad de eliminar la judería y expulsar a lcs judíos de la ciudad en 1396, para evitar la «contaminación» de las almas de los nuevos convertidos, a la vez que se recomendaba a éstos que trasladasen su residencia a otros puntos de la ciudad ${ }^{24}$.

Sin embargo, los conversos ni podían, ni querían renunciar a los vínculos intragrupales para verse perdidos en una maraña de nuevas relaciones incontroladas, por ello tratarán de rearticular las viejas solidaridades.

En primer lugar la práctica de la endogamia parece generalizada durante todo el período estudiado. Tan sólo conocemos un caso en el que pudiera aventurarse la hipótesis de un matrimonio mixto: en 1397, Johan Tamarit, «laborator", vecino de Valencia, dota con 20 libras a Clara, su hija, para que se case con Johan Çapinya, sastre, hijo de Guillem Çapinya ${ }^{25}$. En el documento no aparece en ningún caso la calificación de converso, sin embargo, gracias a su testamento, sabemos que Guillem Çapinya era un neófito. En conclusión, a través de lo que hemos observado

24 Vidal Beltrán, E., op. cit., págs. 51 y ss.

25 ARV, pág. 10407, 1397-septiembre-21. 
en los documentos relacionados con el matrimonio y la dote, pensamos que los conversos practicaron generalmente el matrimonio en el seno del grupo, con tendencia a la isogamia, al menos en los primeros tiempos, de cara a mantener estables los lazos intragrupales y a reforzar relaciones económicas. La hipótesis planteada sería pensar en una reagrupación familiar y, por tanto, patrimonial en torno a los diferentes estatus económicos en los que podemos estratificar al grupo converso.

Los nuevos convertidos buscaron siempre la vecindad. Aunque algunos de ellos, tras el asalto, se trasladaron a otras parroquias, incluso a otras localidades, la mayoría retornaron pronto al antiguo barrio judío. Desde 1396 aparecen con gran frecuencia conversos comprando y alquilando cadas ubicadas en la "òlim juheria vella Valencie». La descripción de las afrontaciones de estas casas que aparece en la documentación, nos revela que en una mayoría de casos las viviendas que la circundan se hallan habitadas por conversos. Veámos un ejemplo, en 1399 Gabriel Dez Cortell, sastre converso, vecino de Valencia, y Dolça, su esposa, venden a Johan de Vera, "ólim Mossé Abenbella", también sastre y vecino de Valencia, una casa situada en la parroquia de Santo Tomás, «in judaria olim Valencie", la cual confronta con las casas de Bonanat Salvador, Johan Valleriola y Rosell de Bellpuig, todos conversos, y con el «açoch" ${ }^{26}$. La tendencia a la reagrupación vecinal no es un caso aislado entre los conversos. Haim Beinart, en su libro sobre los conversos de Ciudad Real, también nos habla de la existencia de barrios habitados en exclusiva por los «anussim» ${ }^{27}$. Y es que la integración grupal, en este caso acentuada por la tradición, se nos aparece como una necesidad fundamental para la supervivencia en la sociedad medieval.

Pero, sin duda alguna, la muestra más destacable de la reconstrucción de las solidaridades internas del grupo converso es la fundación, en 1399, de la Cofradía de «Sent Christòfol» ${ }^{28}$. Sin embargo, la idea de crear una cofradía que vertebre las solidaridades conversas parece ser anterior. Ya en 1397, Guillem Jençor, antes Mossé Vido, deja expresado claramente en sus dos testamentos, ambos dei mismo año, que quiere ser enterrado in fossarie quod conversi sive cristiani novi emere intendent, y en el caso de que a su muerte el citado cementerio aún no hubiese sido construido, pide ser enterrado en el lugar que mejor consideren sus albaceas, pero en cuanto dicho osario se halle listo volo corpus mei translaretur in dicto fossarie novo ${ }^{29}$. Ese mismo año, Guillem Rocha, Pere Lagostera, Johan

26 ARV, pág. 1445, 1399-marzo-4.

27 Beinart, H., Los conversos ante el tribunal de la Inquisición. Barcelona, Riopiedras, 1983.

28 Cf. algunas noticias en GaRcía, A., op. cit.

29 ARV, pág. 10407, 1397-junio-27 y julio-31. 
Rocha, Pere Blanch y otros conversos que compondrán el primer núcleo de la cofradía, nombran procurador a Johan Çamora, también converso, para que apele ante la corte del gobernador y ante quien sea necesario, a fin de evitar que se destruya el cementerio judaico de Valencia quod apud Ruçaffa est, o que al menos se permita el honroso traslado de los difuntos a un lugar apropiado ${ }^{30}$. Esta ordenanza entra dentro de una serie de medidas, algunas de las cuales hemos citado más arriba, que buscan imposibilitar a los conversos cualquier intento de vuelta atrás: la desestructuración de los lazos de solidaridad antiguos. La necesidad de crear un nuevo marco de integración se hace perentoria. A finales de 1398, el platero Martín de Lluna es comisionado por Arnau de Vilarnau y aliquos singulares conversorum dicte civitatis [Valencie] para solicitar ante la corte regia el permiso necesario pro habendo et obtinendo graciam a domino rege ratione constitucionis dicti confratrie beati Christofori ${ }^{31}$. A partir de aquí, las cosas se aceleran, se crea una almoneda (almoina) y comienza a construirse un cementerio. Las actividades religiosas y sociales relacionadas con la cofradía tendrán lugar en la Iglesia de San Cristóbal, construida sobre una antigua sinagoga ${ }^{32}$. La nueva entidad se nutrirá de las donaciones de los conversos valencianos y son numerosos los legados testamentarios que a ella llegan junto con la solicitud de enterramiento en el nuevo cementerio. Arnau de Vilarnau, quizá el más emprendedor de los miembros fundadores, compró unos patios en la parroquia de San Andrés, contiguus orto vocato d'En Vassall, los cuales, tras algunas modificáciones, acogerían el cementerio de los cristianos nuevos, que empezaría a ser usado ese mismo año ${ }^{33}$.

La función de la cofradía estaba clara. No sólo era el nuevo marco receptor de las solidaridades grupales conversas, sino también la pantalla protectora que permitiría el mantenimiento del antiguo culto y las viejas costumbres. Pocos debieron ser aquellos que olvidaron su fe y se hicieron sinceramente cristianos, la gran mayoría de los conversos son criptojudíos $y$, probablemente, judaizantes. La compra de ciertas biblias escritas en caracteres hebreos, el uso de sudarios en los enterramientos conversos, y que la tierra con que se rellenó el nuevo cementerio procediera de Sagunt (probablemente del importante cementerio judío de esta localidad), son indicadores del grado de «cristianización» del «pueblo de los neófitos».

\footnotetext{
ldem, 1397-octubre-31.

ARV, pág. 1445, 1399-febrero-25.

García, A., op. cit., pág. 55.

ARV, pág. 1445, 1399-mayo-26.
} 
Las relaciones con los judíos debieron ser abundantes y en absoluto enfrentadas. Los lazos intrafamiliares entre convertidos y hebreos son continuos y fluidos, tanto en lo económico como en lo social. Se ayudan mutuamente, se asocian y delegan tareas. Con seguridad, los conversos valencianos viajaban a Sagunt varias veces al año, probablemente para adoctrinarse o asistir a los servicios de la sinagoga, a la cual dejaban sustanciosas limosnas ${ }^{34}$. Los hebreos eran conscientes de que las conversiones habían sido impuestas por una sociedad intolerante, y que muchos de sus correligionarios habían abrazado formalmente el cristianismo tan sólo para salvar la vida. Por tanto, como hemos dicho, pensamos que las relaciones entre judíos y conversos a lo largo del 400 valenciano fueron eminentemente próximas y continuas.

En conclusión podemos decir que si bien, a finales del siglo XIV, las familias judeoconversas atraviesan un momento delicado, su situación dista mucho de ser catastrófica. En lugar de abandonarse al pánico, generarán una serie de estrategias que facilitarán la recuperación económica y la reconstrucción de las solidaridades, dentro del nuevo marco surgido del furor antisemita de 1391. Por lo que se refiere a los comportamientos económicos, retomarán sus actividades anteriores implementándolas, y los encontraremos en toclas las esferas de la vida económica, desde las tareas artesanales hasta las relacionadas con la producción y comercialización de los productos agrarios. A lo largo de toda la década ampliarán sus actividades financieras, no sólo a través del préstamo, sino también mediante la adquisición de rentas, procedentes tanto de la compra de censales y violarios, como del arrendamiento de pequeños señoríos pertenecientes a las clases urbanas más elevadas. A partir de 1395 , se evidencia una recuperación sustancial de la economía judeoconversa. Sin embargo, hemos de reseñar que el posicionamiento social y económico del grupo de neófitos queda, todavía, muy alejado del nivel que algunas familias alcanzarán durante el siglo xv.

Socialmente, los conversos dirigirán sus esfuerzos a reconstruir las solidaridades y los patrimonios en el interior de la sociedad cristiana, sin renunciar a sus tradiciones. Practicarán la endogamia dentro de unos niveles de riqueza similares, mantendrán fuertes relaciones con los judíos y seguirán viviendo en comunidad en el interior de la urbe valenciana. Su ascenso social es todavía limitado, la gran mayoría se avecindarán en Valencia y algunos de ellos, los más favorecidos económicamente, adquirirán la categoría de ciudadanos. Fruto de este espíritu de reconstrucción

34 García, A., op. cit., sobre todo caps. II y III. 
y de su incipiente ascenso en el seno de la sociedad cristiana, será la Cofrafía de San Cristóbal. Excluidos de las instituciones de ayuda mutua y de las corporaciones gremiales existentes, la nueva cofradía representará un nuevo marco de fortalecimiento de las relaciones intragrupales, de autodefensa y de mantenimiento de las antiguas tradiciones.

Los conversos valencianos entrarán en el 400 con unas buenas perspectivas de crecimiento económico y ascenso social, favorecidas por el relativo período de tranquilidad de la primera mitad del siglo $\mathrm{XV}$, tras el cual sus expectativas serían cortadas por las actuaciones de la Inquisición. 